\title{
Whole genome capture of vector-borne pathogens from mixed DNA samples: a case study of Borrelia burgdorferi
}

\author{
Giovanna Carpi ${ }^{1{ }^{*} \dagger}$, Katharine S. Walter ${ }^{1 \dagger}$, Stephen J. Bent ${ }^{2}$, Anne Gatewood Hoen ${ }^{3}$, Maria Diuk-Wasser ${ }^{1,4}$ \\ and Adalgisa Caccone $e^{1,5}$
}

\begin{abstract}
Background: Rapid and accurate retrieval of whole genome sequences of human pathogens from disease vectors or animal reservoirs will enable fine-resolution studies of pathogen epidemiological and evolutionary dynamics. However, next generation sequencing technologies have not yet been fully harnessed for the study of vector-borne and zoonotic pathogens, due to the difficulty of obtaining high-quality pathogen sequence data directly from field specimens with a high ratio of host to pathogen DNA.

Results: We addressed this challenge by using custom probes for multiplexed hybrid capture to enrich for and sequence 30 Borrelia burgdorferi genomes from field samples of its arthropod vector. Hybrid capture enabled sequencing of nearly the complete genome ( 99.5\%) of the Borrelia burgdorferi pathogen with 132-fold coverage, and identification of up to 12,291 single nucleotide polymorphisms per genome.

Conclusions: The proprosed culture-independent method enables efficient whole genome capture and sequencing of pathogens directly from arthropod vectors, thus making population genomic study of vector-borne and zoonotic infectious diseases economically feasible and scalable. Furthermore, given the similarities of invertebrate field specimens to other mixed DNA templates characterized by a high ratio of host to pathogen DNA, we discuss the potential applicabilty of hybrid capture for genomic study across diverse study systems.
\end{abstract}

Keywords: Hybrid capture, Whole-genome sequencing, SNPs, Tick-borne pathogens, Lyme disease

\section{Background}

Next generation sequencing (NGS) technologies have transformed infectious disease molecular epidemiology, generating unprecedented amounts of high quality genomic data for a range of downstream applications. The rapidly declining cost, low error rate, and high throughput of existing second-generation platforms enables generation of whole-genome sequences (WGS) for inventory of standing pathogen genomic variation across space and time and development of powerful single nucleotide polymorphism (SNP) markers [1-4]. High density, genome-wide SNPs are critical for fineresolution studies of pathogen transmission chains,

\footnotetext{
* Correspondence: giovanna.carpi@yale.edu

'Equal contributors

'Department of Epidemiology of Microbial Diseases, Yale School of Public Health, 06520 New Haven, CT, USA

Full list of author information is available at the end of the article
}

population structure, phylogeography, phylogenomics, and for a range of genome-wide association studies (GWAS).

However, the power of NGS has not yet been fully harnessed for vector-borne and zoonotic disease (VBZ) systems, the majority of globally emerging infectious diseases [5]. Although NGS sequencing has provided valuable insight into the genomic diversity of easily culturable VBZ parasites such as Plasmodium spp. and Trypanosoma spp.[6-10], large-scale genomic studies of field-collected pathogens has been stymied by the difficulty of obtaining high-quality WGS data for pathogens more difficult to culture. This is due to the high ratio of host (the arthropod vector or the reservoir host) to pathogen DNA present in mixed DNA samples [11, 12]. The overwhelming presence of this exogenous DNA (which may also include commensal bacteria, 
endosymbionts, and environmental microbes) renders shotgun sequencing inefficient and cost-prohibitive. The development and availability of an efficient, and cost-effective method to retrieve WGS directly from field-collected VBZ samples will (1) enable study of pathogen demographic and evolutionary history, providing critical information to predict pathogen spread or monitor the success for epidemiological interventions, and (2) provide a wealth of genomic resources for association studies [13].

The agent of the most prevalent vector-borne disease in the US, the Lyme disease bacterium, Borrelia burgdorferi sensu stricto, exemplifies this common hurdle to genomic study for many VBZ systems. B. burgdorferi's small genome $(\sim 1.5 \mathrm{Mb})$, is overwhelmed by the genome size of its black-legged tick vector, Ixodes scapularis ( 2.1Gb) [14-17] and, in average field collected nymphal ticks, B. burgdorferi represents $<0.01 \%$ of the total DNA template. Pure B. burgdorferi cultures would be the ideal template for generation of genomic DNA libraries, enabling maximally efficient deep sequencing. However, culturing B. burgdorferi directly from its tick vector is labor intensive and culture success is strain-dependent, introducing significant bias [18]. Shotgun approaches for WGS of pathogens directly from field samples are inefficient due to the excess of contaminating host DNA present in the samples, which result in very low sequence coverage of the low copy number bacterial genomes present [11]. Recently, the use of selective whole genome amplification to amplify DNA from the target bacteria from mixed DNA samples has been proposed [19]. While this method enables enrichment of B. burgdorferi DNA from artificial mixtures of bacterial DNA, it was not validated for $B$. burgdorferi genome sequencing directly from field-collected ticks with spirochete burdens expected in natural infections. Furthermore, this method includes a PCR-based whole genome amplification step prior to sequencing, introducing several potential biases [20-22].

Despite the well documented increase in incidence of human Lyme disease over the last forty years [23], B. burgdorferi molecular epidemiology and population genetic studies have relied on analyses of single genes or multi-locus sequence typing based on eight housekeeping genes (MLST) [24-27]. Use of these coarse markers precludes fine-scale study of B. burgdorferi variation in the field and highlights the need for a population genomics approach for study of the epidemiology and biology of this important human pathogen.

The advent of hybrid capture (or hybrid enrichment) in synergy with NGS has overcome limitations related to low input and mixed DNA samples [21, 28, 29]. Using custom oligonucleotide arrays, hybrid capture harnesses the differential power of DNA hybridization to extract a genome of interest from a mixed template. This method was originally developed for human genome-wide association studies [28], where it was used to selectively resequence the human exome [29]. Hybrid capture does not require amplification prior to sequencing, thus circumventing the biases known to be introduced by whole genome amplification and similar methods [21, 22, 30]. Moreover, hybrid capture can enrich for divergent sequences, even when oligonucleotide arrays used to capture the targeted genome are derived from a single reference genome [31, 32]. Although this method has been successfully applied in a variety of systems $[21,29$, 31-34] from ancient human DNA [35] to modern human clinical samples of Plasmodium falciparum [32], it has not yet been exploited for assessing pathogen genomic diversity directly from mixed DNA template specimens derived from arthropod vectors and/or animal reservoir hosts.

In this study, we demonstrate the efficiency of combining hybrid capture techniques and NGS to enrich for B. burgdorferi genomic DNA directly from field collected vector samples. Using this combined approach we $i$ ) evaluate the capture efficiency for B. burgdorferi genomes in different multiplexed capture strategies, $i$ ) generate $30 \mathrm{~B}$. burgdorferi WGS at high coverage, and iii) identify high quality genome-wide SNPs. This new efficient and cost-effective method can be readily applied to a variety of VBZ disease systems and enables us to take advantage of field collections for population genomic studies of emerging pathogens.

\section{Results}

\section{Whole genome capture efficiency}

\section{Shotgun sequencing}

Using one Hiseq2000 lane, we directly sequenced two whole tick DNA extracts (samples Sh834 and Sh1589, Table 1 ) with a $B$. burgdorferi load, measured by qPCR, within the expected range of field-collected nymphs (3721 and 1184 spirochete copies, respectively) [36]. This generated $\sim 144$ million reads (75 bp paired end reads) with a mean capture efficiency (the proportion of sequence reads mapping to our target of interest, the $B$. burgdorferi B31 reference genome) of $<0.01 \%$ (Table 1 ). The majority of remaining sequence reads (average: $66.49 \%)$ mapped to the tick vector I. scapularis draft assembly. This represents an underestimate of tick-derived reads, as the $\sim 66 \%$ of the I. scapularis draft assembly consists of highly repetitive regions affecting the overall mapping efficiency [37-39].

\section{Target capture and sequencing}

We performed whole genome capture using custom $B$. burgdorferi probes of 30 unique field-collected infected 
Table 1 Mapping statistics. For each sample the first four columns list the sample names followed by the capture reaction (number of genomic libraries captured in multiplex), the total number of sequenced bases (Mb) prior to filtering, and the percentage PCR duplicates. The final five columns report the capture efficiency (percentage of reads that mapped to the B. burgdorferi B31 reference genome), the percentage of sequence covered by at least one generated sequence read and the fold coverage (the total number of unique sequence reads which map to each nucleotide in the reference B31 genome) of filtered data for the linear chromosome $(911 \mathrm{~Kb})$ and the longest linear plasmid, Ip54 $(53 \mathrm{~Kb})$, respectively

\begin{tabular}{|c|c|c|c|c|c|c|c|c|}
\hline Sample & $\begin{array}{l}\text { Capture } \\
\text { reaction }\end{array}$ & $\begin{array}{l}\text { Sequenced } \\
\text { bases }(\mathrm{Mb})\end{array}$ & $\%$ duplicate & $\begin{array}{l}\text { Capture } \\
\text { efficiency }\end{array}$ & $\begin{array}{l}\% \text { Chr } \\
\text { covered }\end{array}$ & $\begin{array}{l}\text { Coverage } \\
\text { depth (Chr) }\end{array}$ & $\begin{array}{l}\% \text { Ip54 } \\
\text { covered }\end{array}$ & $\begin{array}{l}\text { Coverage } \\
\text { depth (Ip54) }\end{array}$ \\
\hline Bbcap1 & 20 & 34.22 & 42.63 & 0.44 & 99 & 11.71 & 98 & 12.77 \\
\hline Bbcap2_L1 ${ }^{a}$ & 10 & 108.45 & 50.1 & 0.64 & 100 & 52.92 & 100 & 52.53 \\
\hline Bbcap2_L2 ${ }^{\text {b }}$ & 4 & 237.68 & 70.49 & 0.61 & 100 & 109.52 & 100 & 113.51 \\
\hline Bbcap3 & 20 & 94.32 & 56.52 & 0.76 & 100 & 52.6 & 100 & 59.7 \\
\hline Bbcap4_L1 & 1 & 2066.79 & 92.57 & 0.10 & 100 & 140.83 & 98 & 194.75 \\
\hline Bbcap4_L2 & 4 & 188.60 & 61.81 & 0.56 & 100 & 76.92 & 100 & 94.68 \\
\hline Bbcap5 & 20 & 163.28 & 61.93 & 0.81 & 100 & 109.28 & 100 & 66.03 \\
\hline Bbcap6_L1 & 10 & 334.98 & 69.68 & 0.81 & 100 & 217.77 & 98 & 208.67 \\
\hline Bbcap6_L2 & 20 & 202.09 & 64.25 & 0.81 & 100 & 131.54 & 98 & 125.66 \\
\hline Bbcap7 & 20 & 75.67 & 55.01 & 0.65 & 100 & 39.08 & 98 & 34.93 \\
\hline Bbcap8 & 20 & 41.28 & 47.49 & 0.49 & 100 & 13.87 & 100 & 18.71 \\
\hline Bbcap9 & 10 & 221.18 & 67.38 & 0.76 & 99 & 134.32 & 98 & 148.14 \\
\hline Bbcap10 & 20 & 449.34 & 81.21 & 0.91 & 100 & 305.33 & 100 & 259 \\
\hline Bbcap12 & 20 & 185.23 & 67.57 & 0.77 & 100 & 103.49 & 98 & 91.28 \\
\hline Bbcap13 & 10 & 617.48 & 83.63 & 0.81 & 100 & 331.14 & 98 & 327.07 \\
\hline Bbcap14 & 20 & 140.75 & 62.89 & 0.70 & 100 & 76.99 & 98 & 86.69 \\
\hline Bbcap15 & 10 & 69.67 & 49.41 & 0.43 & 99 & 23.64 & 99 & 33.1 \\
\hline Bbcap16 & 20 & 119.57 & 54.98 & 0.72 & 99 & 71.59 & 99 & 71.73 \\
\hline Bbcap17 & 20 & 344.88 & 72.98 & 0.84 & 100 & 219.8 & 100 & 245.4 \\
\hline Bbcap19 & 10 & 53.31 & 41.46 & 0.53 & 100 & 19.73 & 100 & 25.17 \\
\hline Bbcap20 & 20 & 128.15 & 59.07 & 0.72 & 100 & 72.48 & 100 & 84.78 \\
\hline Bbcap21 & 20 & 135.54 & 60.87 & 0.71 & 99 & 72.32 & 98 & 79.68 \\
\hline Bbcap22 & 4 & 976.61 & 91.94 & 0.88 & 100 & 597.31 & 100 & 782.62 \\
\hline Bbcap23 & 20 & 159.50 & 63.51 & 0.64 & 99 & 77.6 & 98 & 105.44 \\
\hline Bbcap24 & 20 & 84.34 & 49.5 & 0.65 & 100 & 42.99 & 100 & 29.66 \\
\hline Bbcap25 & 20 & 61.60 & 50.95 & 0.50 & 99 & 25.13 & 98 & 28.68 \\
\hline Bbcap26 & 20 & 75.92 & 57.04 & 0.53 & 99 & 30.63 & 98 & 31.59 \\
\hline Bbcap27 & 20 & 73.49 & 60.97 & 0.59 & 100 & 33.45 & 100 & 35.05 \\
\hline Bbcap28 & 20 & 123.64 & 65.07 & 0.73 & 100 & 72.31 & 99 & 61.74 \\
\hline Bbcap29 & 10 & 255.63 & 72.17 & 0.86 & 100 & 167.46 & 100 & 178.06 \\
\hline Bbcap30_L1 & 10 & 343.08 & 70.18 & 0.83 & 100 & 225.25 & 99 & 183.62 \\
\hline Bbcap30_L2 & 4 & 825.19 & 88.1 & 0.79 & 100 & 520.17 & 100 & 438.71 \\
\hline Bbcap31_L1 & 10 & 292.77 & 65.76 & 0.85 & 100 & 198.23 & 100 & 136.51 \\
\hline Bbcap31_L2 & 20 & 232.33 & 66.08 & 0.85 & 100 & 156.58 & 100 & 109.34 \\
\hline Bbcap32 & 10 & 164.70 & 60.74 & 0.74 & 99 & 87.35 & 98 & 104.07 \\
\hline Sh834 ${ }^{c}$ & Shotgun & 9954.53 & 19.92 & $6.00 \mathrm{E}-05$ & 7.7 & 1.2 & 0.8 & 1.23 \\
\hline Sh1589 & Shotgun & 825.75 & 18.15 & 1.40E-04 & 27.1 & 1.389 & 38 & 1.5 \\
\hline
\end{tabular}

a L1: Samples (indexed genomic libraries after capture) that were sequenced in a half lane

${ }^{b}$ L2: Samples (indexed genomic libraries after capture) that were sequenced in a different half lane

'Sh: samples sequenced using shotgun approach 
tick samples. These samples were previously genotyped and include 14 MLST sequence types and 9 IGS types (Additional file 1: Table S1). The spirochete load for all the analyzed samples, measured by qPCR, fell within the expected range of field-collected nymphs (median = 784 genome equivalents, range 157-3156 copies) [36] (Table 1). Sample DNA content was a conservative estimate of the range of DNA yield within field-collected nymphal ticks extracted through standard procedures $($ mean $=111 \mathrm{ng}$, range, 53-189 ng) (Table 1).

The hybridization capture approach effectively enriched for $B$. burgdorferi in all examined genomic samples. The thirty-five enriched samples (30 field tick samples and five replicates used in the different multiplex schemes, see Methods section and Additional file 1: Figure S1) were pooled and sequenced on one Illumina HiSeq 2500 lane, generating 9.7 Gigabases $(\mathrm{Gb})$ of sequence data. After PCR and optical duplicates were removed, we obtained an average of 276.6 Megabases $(\mathrm{Mb})$ of read data/sample (range: 34.2-2066 Mb), of which a mean of $175.1 \mathrm{Mb}(15.2-859.1 \mathrm{Mb})$ mapped to the $B$. burgdorferi B31 reference genome. Mean capture efficiency across samples was $68.6 \%$ (range 9.59$90.58 \%$ ) (Table 1, Additional file 1: Figure S2). On average, $62.7 \mathrm{Mb} /$ sample (11.9-1388 Mb) mapped to the $I$. scapularis draft assembly ( $10.1 \%$ of all sequence reads; $2.05 \%-25.3 \%)$. Whole genome capture efficiency of $B$. burgdorferi was significantly correlated with proportion of tick-derived reads captured (Additional file 1: Figure S3A) $(\mathrm{x} 2=1.21, \mathrm{p}<0.001)$ and with the B. burgdorferi input sample load (Additional file 1: Figure S3B $)(x 2=0.376, p<0.001)$ in univariate models.

\section{Whole genome coverage}

Coverage statistics were calculated for the entire B. burgdorferi B31 reference genome $(1.52 \mathrm{Mb})$, including the linear chromosome $(911 \mathrm{~Kb})$ and 21 plasmids $(5-53 \mathrm{~Kb})$.

\section{Shotgun sequencing}

Across the two samples, $17.4 \%$ of the B. burgdorferi chromosome was covered at an average coverage of 1.3X. Similarly low coverage was obtained for the longest plasmid lp54 $(53 \mathrm{~Kb})$, with $23 \%$ of the sequence covered at 1.3X (Table 1).

\section{Target capture and sequencing}

Across the 30 unique samples and the five replicates, $99.5 \%$ (range: 98.7-99.7 \%) of the chromosome was covered at an average coverage of 132X (range: 11.7597X) (Table 1, Fig. 1a). Two short chromosomal regions between 435-438 $\mathrm{Kb}$ and 438-444 $\mathrm{Kb}$ exhibited low coverage across all samples (Fig. 1a). These regions contain the $23 \mathrm{~S}$ rRNA duplicated genes [40]. For the longest plasmid, lp54, the average portion covered was
98.9 \% (range: 97.6-100 \%) at 133X (range: 12.8782.6X) (Table 1, Fig. 1b). A lower coverage was observed across the other 9 circular plasmids and the 11 linear plasmids, with $62 \%$ of the plasmid sequences covered at an average of 63 -fold coverage, and with $75 \%$ of the plasmid sequences at an average of 80 -fold coverage, respectively (Additional file 2 : Table S2A and B). Among the circular plasmids, cp32-1 and cp32-8 showed the lowest percentage bases covered (on average $20 \%$ of covered bases at 27 -fold coverage and $28 \%$ at 37 -fold coverage) (Additional file 2: Table S2A), and among the linear plasmids, lp5 was the only linear sequence showing less than $50 \%$ of covered bases, at an average of 45 fold coverage (Additional file 2: Table S2B).

\section{Comparison of multiplexing schemes}

We assessed the efficiency of capturing B. burgdorferi when multiplexing different numbers of samples for hybrid capture reaction. The multiplexing strategy is schematically depicted in Additional file 1: Figure S1. Additional file 1: Figure S2 shows the results of the hybrid capture by multiplexing 1, 4, 10 and 20 indexed tick genomic DNA libraries in a single pool. Table 1 summarizes $B$. burgdorferi capture efficiency for each sample by capture reaction. High average capture efficiency for the 4-plex, 10-plex, and 20-plex captures was consistently achieved (median: $70.3 \%, 78.2 \%$, and $71.5 \%$ ), whereas the 1-plex capture sample efficiency was low (10\%). The logistic regression models applied to correlate capture efficiency with the proportion of tick-derived reads and B. burgdorferi input sample load, identified the sample captured in 1-plex as a significant outlier (see Methods). We speculate that the 1-plex sample presented low efficiency due to the possible saturation of capture probes by non-specific DNA template molecules. However, we do not have replicates of the 1-plex capture and the low efficiency may reflect the stochasticity of capture.

Per sample chromosomal coverage plots for the ten samples captured in the 10-plex capture reaction, which illustrate the variation expected in coverage among multiple samples captured together, are depicted in Additional file 1: Figure S4.

Five tick samples were captured in duplicate, allowing for direct comparisons of capture efficiency for the same starting genomic library and pathogen load. Table 2 compares capture efficiency among sample replicates. Although the total number of reads generated per sample was inversely correlated with the number of samples pooled, no significant changes in efficiency between captures was observed, except for the 1-plex capture, a clear outlier with significantly lower efficiency than the other captures (Tables 1 and 2, Additional file 1: Figure S3A, B.) 


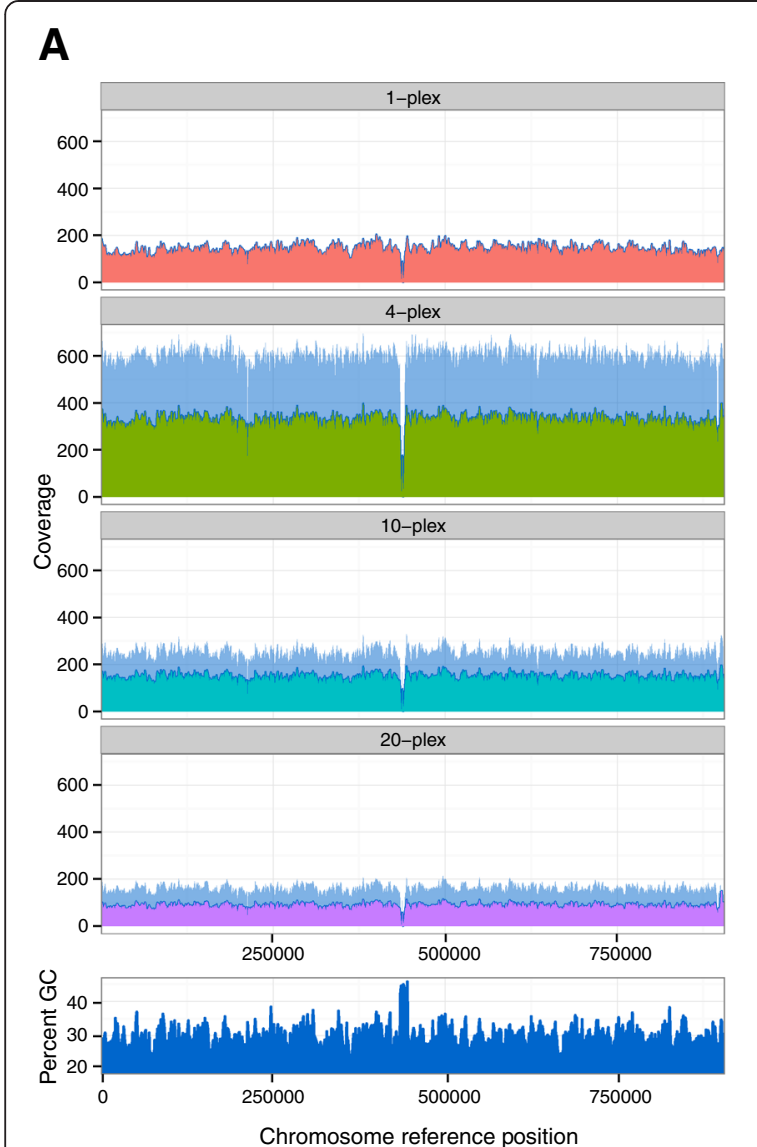

B

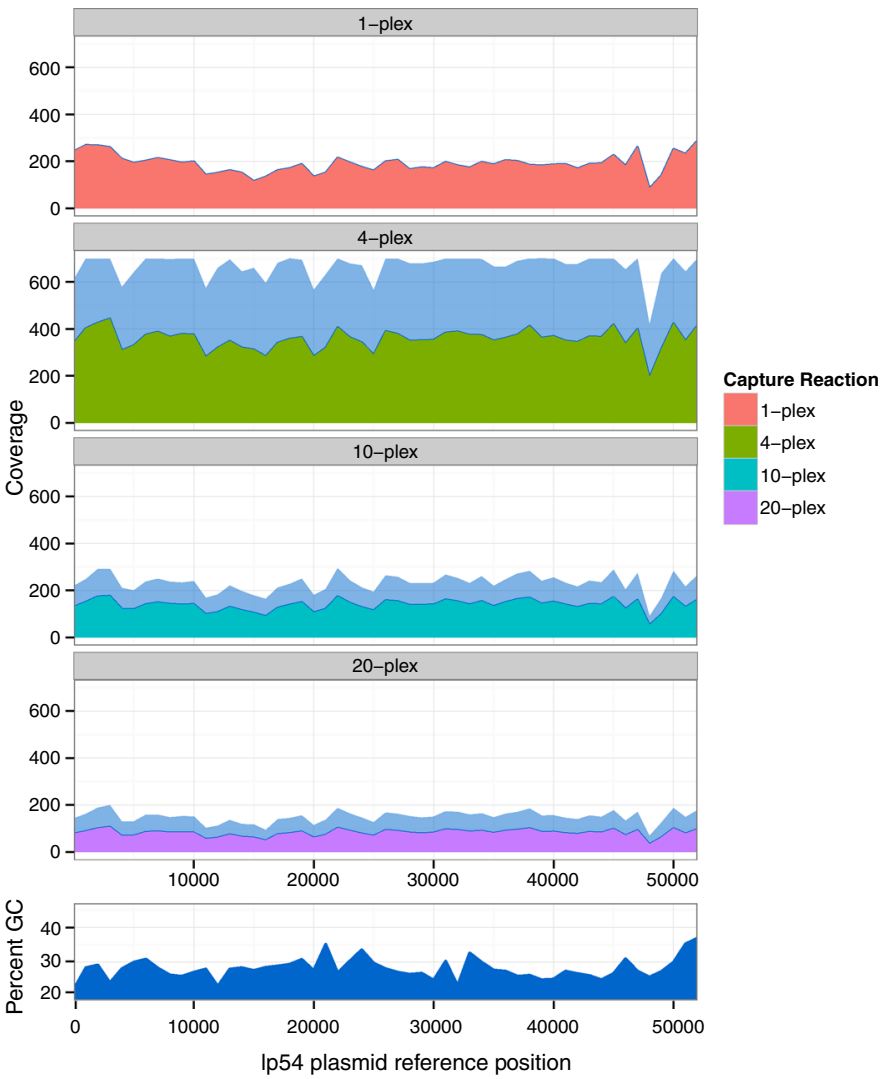

Fig. 1 Comparison of the mean coverage for B. burgdorferi genomes across capture reactions. a The linear chromosome (910,724 bp) and b the linear plasmid, Ip54 (53,657 bp). The y-axis indicates the coverage depth averaged over all samples in each capture reaction, defined as the total number of sequenced bases which map to each nucleotide in the reference B31 genome calculated on a window size of 1000 bp after removal of potential PCR duplicates and reads with mapping quality $\leq 20$. The $x$-axis indicates the reference position relative to the samples; the light blue region indicates the area within one standard deviation of the mean coverage of samples captured in the capture reaction. GC content (percentage of nucleotide in the genome that are $\mathrm{G}$ or C) for the chromosome and the linear plasmid calculated on a 1000 bp window size are shown below the coverage plots

\section{Genome-wide SNPs and SNP effect analysis}

We detected 262,987 nucleotide polymorphic sites for the entire data set, with a range of 437-12,291/genome. An average of 4055 SNPs (range: 49-7126) were randomly distributed across the B. burgdorferi B31 chromosome ( 1 SNP per $227 \mathrm{bp}$ ) (Additional file 1: Figure S5). The program SnpEff v2.0b was used to predict the effect of the identified SNPs on B. burgdorferi coding sequence. For this analysis, we focused on the chromosome as plasmids contain large stretches of DNA rearrangements, non-homologous recombinations, and inverted repeats. On average 3670 SNPs were found in coding sequences (CDS), of which 1203 were classified as non-synonymous mutations (Additional file 1: Table S3 and S4). The average ratio of non-synonymous to synonymous (N/S) mutations was 0.49 (not normalized). Pairwise comparison of identified SNPs and number of SNPs within CDS between replicate samples showed consistency in SNP detection and location of SNPs across capture pools (Additional file 1: Table S4).

\section{Discussion}

Our results demonstrate the feasibility of combining hybrid capture techniques with NGS to successfully enrich for a target pathogen, B. burgdorferi, directly from fieldcollected vector samples. Using this approach, we generated high quality genomic resources and a repository of genetic variants for 30 unique $B$. burgdorferi field strains for a variety of downstream applications. We obtained high capture efficiency $(66.49 \%)$ and nearly complete coverage of the B. burgdorferi genome (99.5\%) at 132fold coverage across all the 35 analyzed samples. By contrast, shotgun sequencing with no pre-enrichment step was significantly less efficient, resulting in only $0.01 \%$ of B. burgdorferi-derived sequences (Table 1). The low efficiency of the shotgun approach is consistent with 
Table 2 Pairwise comparison of multiplex captures. Comparison of B. burgdorferi capture efficiency from tick genomic libraries that were captured twice in different reaction capture using different multiplexing scheme

\begin{tabular}{|c|c|c|c|c|c|c|}
\hline Sample & $\begin{array}{l}\text { No. pooled } \\
\text { samples }^{\text {a }}\end{array}$ & Total reads (bp) & $\begin{array}{l}\text { B. burgdorferi mapped } \\
\text { reads (bp) }\end{array}$ & $\begin{array}{l}\text { B. burgdorferi } \\
\text { efficiency }^{c}\end{array}$ & $\begin{array}{l}\text { Tick mapped } \\
\text { reads (bp) }{ }^{d}\end{array}$ & $\begin{array}{l}\text { Tick } \\
\text { efficiency }\end{array}$ \\
\hline \multirow[t]{2}{*}{ Bbcap4 } & 1 & $27,557,241$ & $2,644,289$ & 0.10 & $6,032,190$ & 0.15 \\
\hline & 4 & $2,514,653$ & $1,399,218$ & 0.56 & 503,015 & 0.14 \\
\hline \multirow[t]{2}{*}{ Bbcap2 } & 4 & $3,169,012$ & $1,939,221$ & 0.61 & 616,781 & 0.12 \\
\hline & 10 & $1,445,990$ & 927,938 & 0.64 & 281,094 & 0.15 \\
\hline \multirow[t]{2}{*}{ Bbcap30 } & 4 & $11,002,588$ & $8,744,400$ & 0.79 & 582,618 & 0.03 \\
\hline & 10 & $4,574,392$ & $3,780,942$ & 0.83 & 244,753 & 0.04 \\
\hline \multirow[t]{2}{*}{ Bbcap31 } & 10 & $3,903,612$ & $3,328,957$ & 0.85 & 269,657 & 0.05 \\
\hline & 20 & $3,097,722$ & $2,638,052$ & 0.85 & 199,022 & 0.05 \\
\hline \multirow[t]{2}{*}{ Bbcap6 } & 10 & $4,466,415$ & $3,605,584$ & 0.81 & 260,144 & 0.04 \\
\hline & 20 & $2,694,537$ & $2,190,349$ & 0.81 & 136,170 & 0.04 \\
\hline
\end{tabular}

${ }^{a}$ Number of sample mutliplexed in a single capture reaction

${ }^{\mathrm{b}}$ Total number of sequenced bases mapped to the $B$. burgdorferi B31 reference genome after removal of PCR and optical duplicates

'Proportion of sequences that mapped to the reference genome after removal of PCR and optical duplicates

${ }^{\mathrm{d}}$ Total number of sequenced bases mapped to the $I$.scapularis draft assembly $[16,17]$ after removal of PCR and optical duplicates

previous findings using the same approach directly on infected ticks, where on average only $1 / 3$ of $B$. burgdorferi genome/sample was recovered [11].

Obtaining high-quality reads with even coverage of the reference can be difficult for templates with biased nucleotide composition [41]. However, we achieved consistently uniform coverage along the majority of the $B$. burgdorferi chromosome (Fig. 1a), although its nucleotide composition is AT-rich (71\%), suggesting that even low GC content microbial genomes can be efficiently sequenced with this method. The incomplete coverage of some plasmids (Additional file 2: Table S2A and B) is expected as previous studies have found DNA rearrangements and inverted repeats are common across $B$. burgdorferi plasmids [42], rendering mapping difficult with short-read data alone.

Hybrid capture avoids both bacterial culture and whole genome amplification, currently standard methods used to enrich pathogens prior genome sequencing and known to be biased in strain representation and propagation of sequencing errors, respectively [18, 20-22]. Further, our study validates this approach for fieldcollected samples representative of the B. burgdorferi copy number and total arthropod DNA content found in natural vector populations.

In the 30 WGS, we identified high levels of genetic polymorphisms at the chromosomal level (49-7126 SNPs per sample). This is a significant improvement from the coarse resolution provided by the highly-conserved MLST markers [26], previously considered the gold-standard for $B$. burgdorferi molecular typing, and other common markers such as IGS and $O s p C[26,43]$.

Comparison of different multiplexed capture pools demonstrates high capture efficiency and genome coverage of
B. burgdorferi even when multiplexing up to 20 genomic libraries in a single capture reaction (Tables 1 and 2), with equivalent sensitivity in SNP detection observed in the pairwise comparison of the replicates in the 10-plex or 20plex reaction (Additional file 1: Table S4). Thus, this multiplexed capture method is highly scalable, substantially lowering the capture reaction cost per sample.

Many other VBZ pathogens currently share the same research bottleneck: the difficulty in obtaining a sufficient number of pathogen genomes from suboptimal field samples for population genomic study. Hybrid capture is generalizable and can be used to study both VBZ and other pathogens avoiding the difficulties, biases, and costs inherent to pathogen culture or whole genome amplification. Multiple infections involving multiple parasite species within a single vector specimen as well as mixed infections involving genetically distinct clones of the same parasite species within a single vector are frequent [44]. In the first instance, hybrid capture methods may be applied for population genomic study of several bacterial, protozoan, or viral pathogens co-vectored by the same blacklegged tick vector such as Anaplasma phagocytophilum, Babesia microti, and Powassan virus [45-49] using a multiple-pathogen capture array. In the second case, hybrid capture can enrich for the entire population of a single pathogen infecting a single vector or host. If multiple haplotypes are present within the enriched sample, computational and statistical methods can be used to detect and resolve the composition of mixed infections, a level of pathogen diversity often ignored when using pathogen cultures or multi-locus genotyping with Sanger sequencing $[25,44,50,51]$.

This methodology can also be applied across diverse VBZ systems such as study of African trypanosomes 
directly from collections of the tsetse fly [52-54] or American trypanosomes directly from the triatomine vector [55]. Finally, we note that the development of a single set of capture probes for a pathogen of interest will enable generation of pathogen WGS directly from vector and host samples (whether human, animal reservoir, or both), enabling study of host specificity and potential transmission bottlenecks.

Additionally, hybrid capture has a variety of other potential applications in public health and molecular ecology for studies in which the target DNA represents $1 \%$ or less of a mixed DNA sample. For example, hybrid capture could be adopted as a non-invasive method for whole genome sequencing of target pathogens directly from fecal samples from human and wildlife species [56-58], archived human and animal samples that have been fixed or frozen, or from environmental samples [59], enabling early identification of known zoonotic pathogen outbreaks and their potential origins.

Hybrid capture methods will allow for highly efficient and massively parallel re-sequencing of genomes of interest for non-model organisms, while minimizing cost and effort of sequencing host DNA. Given the high capture efficiency observed in this study, the average throughput of a HiSeq 2500 lane passing quality control (25.5 Gbp for paired-end $75 \mathrm{bp}$ reads), and length of our target genome $(\sim 1.52 \mathrm{Mb})$, we determine that sequencing of up to 160 whole B. burgdorferi genomes to an optimal coverage of $30 \mathrm{X}$ can be conservatively conducted on a single Illumina HiSeq2500 lane (requiring only $60 \%$ of a single Illumina lane) and costing approximately $\$ 1887$ (current in-house cost of a single Illumina HiSeq 2500 lane). The cost of hybrid capture and sequencing for a single pathogen with similar genome size to our study organism, for an equivalent coverage, is approximately $\$ 227 /$ per sample when multiplexing at least 10 samples per hybridization capture. This cost estimate includes the costs for the library creation, the synthesis of capture array and the Illumina sequencing (Additional file 1: Table S5). Thus, the efficiency and scalability of the proposed method renders population genomic study of $B$. burgdorferi economically feasible. By contrast, shotgun sequencing of $B$. burgdorferi directly from its vector is highly inefficient, as we obtained only $17.4 \%$ of the pathogen genome at low coverage. To obtain a comparable genome coverage necessary for population genomic study (30X) [60], shotgun sequencing of a single B. burgdorferi-infected tick sample would require 75 HiSeq2500 lanes (Additional file 1: Table S5). The equivalent sequencing coverage for a single hybrid capture sample would require a fraction of the shotgun sequencing effort ( $0.006 \%$ of one shotgun sequencing sample).

We propose that multiplexed hybrid capture can be widely applied to sequence other bacterial, protozoan, and viral genomes that exist in low titers in a variety of mixed DNA samples and will enable fine-resolution population genomic study. Yet, several possible limitations need to be considered. First, a preexisting reference genome for the target pathogen or a closely related species is required for capture array design. However, because probe oligonucleotides can hybridize DNA sequences with up to $78 \%$ nucleotide divergence, probes designed using genomes from closely related species can still efficiently enrich for the target pathogen [31, 61]. Conversely, host reference genomes are not necessary for capture array design. In our study, we took advantage of the available draft genome of the $I$. scapularis tick species to test the efficiency of capture. Second, repetitive elements and recombination events within the target genome may be difficult to resolve. However, our results show that we captured nearly the complete B. burgdorferi genome, on average $99.74 \%$ of the chromosome and $99.09 \%$ of the longest plasmid, lp54, even after excluding repetitive elements that could not be resolved by read mapping alone (Table 1). Given the nature of the genomic data retrieved, it is impossible to identify recombination events. Exclusion of repetitive elements and detailed mapping of recombination events will not preclude evolutionary studies which focus on the large amount of variation present across the rest of the pathogen genome. Finally, captured variation is limited by the set of probes included in the capture array and the sequence divergence allowed (see above), such that novel gene sequences within the target genome may not be captured. However, previous studies have shown that the rate of gene acquisition in B. burgdorferi is higher between than within species, and that this rate is among the lowest in bacterial pathogens [62]. This suggests high genome stability and few lineage-specific genes in $B$. burgdorferi, which reduces the impact of this possible bias in this study system.

\section{Conclusions}

Genomic studies of vector-borne and zoonotic pathogens, such as B. burgdorferi, have been hindered by the high ratio of host-to-pathogen DNA in arthropod vectors. We adapted target enrichment methods for study of the tickborne spirochete $B$. burgdorferi directly from field-derived tick samples. Sequence data enable powerful reconstruction of pathogen transmission chains, phylogenies, and GWAS for cultivable pathogenic microbes. The proposed multiplexed hybrid capture and sequencing method enabled the generation of nearly complete genomes of $B$. burgdorferi and the identification of high density, genomewide SNPs directly from vector samples. We showed that this methodology is highly scalable and cost-effective and can be applied to a variety of VBZ systems as well as broader applications in molecular ecology. 


\section{Methods}

\section{Sample collection and selection}

Field ticks used in this study were collected in 2007 using a standard dragging methodology [63] and preserved in $70 \%$ ethanol. The 30 B. burgdorferi-infected host-seeking I. scapularis nymphs used in this study were chosen to maximize the geographic and pathogen genetic diversity in Northeastern USA. Samples were collected in eight sites in New York, Connecticut, Massachusetts, and Vermont (Additional file 1: Table S1). The two tick specimens used for whole-tick shotgun sequencing were collected using the same methodology in B. burgdorferi endemic sites in Connecticut in June 2012 (Additional file 1: Table S1).

Host-seeking nymphs were identified morphologically as I. scapularis, using standard taxonomic keys [64], and confirmed by PCR amplification and DNA sequencing of the 16S ribosomal RNA (rRNA) gene [65] (data not shown).

After DNA extraction from individual ticks [66], quantitation and quality assessment was carried out using the dsDNA HS Assay on a Qubit Fluorometer (Invitrogen) and Agilent Bioanalyzer 2100 instrument, respectively. Each tick sample was tested in duplicate for B. burgdorferi infection and load using a TaqMan qPCR assay targeting a $68 \mathrm{bp}$ fragment of the $16 \mathrm{~S}$ rRNA gene [67].

\section{Genomic library preparation}

Illumina library preparation, hybridization capture, and sequencing was conducted at the Yale Center for Genomic Analysis (YCGA). Library preparation was conducted using a modified Roche/Nimblegen SeqCap EZ Library Short Read protocol [68]. Library concentration was determined using PicoGreen assay (Invitrogen) and size selection was performed on a Caliper LabChip GX instrument (PerkinElmer).

\section{Capture array design}

A custom targeted sequence capture array for B. burgdorferi was generated using the Roche NimbleGen SeqCap method (Madison, USA) [31, 69], GC-balanced, biotinylated DNA probes were designed in silico to tile $99.7 \%$ of the B. burgdorferi B31 reference genome (GenBank: GCF_000008685.2, 1 linear chromosome and 21 plasmids) $[14,15]$. This isolate is derived from a tick from Shelter Island, NY, USA, the existing reference most geographically and ecologically representative of our samples. We supplemented this B. burgdorferi probe set with probes tiling 128 published IGS haplotypes, and 32 known diverse $\operatorname{sp} C$ haplotypes [70] because these regions are highly divergent across $B$. burgdorderi strains, and because of the role of $\operatorname{ssp} C$ role in transmission [71-73], in addition to being commonly used as markers in population genetic studies $[26,74,75]$. To remove the probes that hybridized to the tick vector, they were screened against the Ixodes scapularis draft assembly IscaW1 (Wikel strain), downloaded from VectorBase $[16,17]$.

\section{Multiplexed whole genome capture and sequencing}

To optimize the multiplexing strategy for maximum $B$. burgdorferi genome capture efficiency, indexed genomic tick libraries were combined in 1, 4, 10, and 20-plex pools. In addition, five samples were captured twice, in two separate multiplexed pools to compare capture efficiency and SNP detection for the same initial B. burgdorferi-load and gDNA library captured in different multiplex reactions (Additional file 1: Figure S1). Equimolar amounts of each indexed genomic tick library were pooled prior to capture for a total of 1 ug total genomic DNA per hybridization reaction, according to the multiplexing strategy described above. Samples were heat-denatured and mixed with the custom DNA probes (Roche/NimbleGen) and hybridization performed at $47^{\circ} \mathrm{C}$ for $68 \mathrm{~h}$. Samples were then washed with a series of stringent buffers to remove non-specifically bound DNA fragments. The captured fragments were PCR amplified and purified with AMPure $\mathrm{XP}$ beads. Sample concentrations were normalized to 2 $\mathrm{nM}$ and loaded onto two half lanes of Illumina version 3 flow cells at a concentration that yields 170-200 million passing filter clusters per lane. Samples were sequenced using 75 bp paired end sequencing on an Illumina HiSeq 2500 at Yale Center for Genomic Analysis (YCGA) according to Illumina protocols. Sample de-multiplexing was performed using Illumina's CASAVA 1.8.2 software suite. Metadata and sequence data of each sample in this study are available in the NCBI under BioProject accession number PRJNA264068.

\section{Shotgun sequencing}

For comparison of genome capture efficiency, DNA shotgun sequencing was conducted on two tick samples: Sh1589 and Sh834 (Additional file 1: Table S1). Sample preparation and genomic library preparation was conducted as described without the hybrid capture step. Genomic libraries were prepared from the two whole tick DNA extracts and sequenced directly on one Illumina HiSeq 2000 lane. The mapping pipeline was identical for both shotgun and captured samples.

\section{Read mapping, genome-wide SNP detection and SNP effect analysis}

Raw Illumina sequence reads for each sample were aligned to the $B$. burgdorferi reference genome (strain B31; [14, 15]) using BWA (v. 0.7.7) [76] with soft clipping of bases with Phred quality score below 20. Duplicate sequence reads were marked using the Picard Suite (v. 1.117) MarkDuplicates (http://broadinstitute.github.io/picard/). The percentage of the bacterial reference 
genome covered (linear chromosome and plasmids), the genome coverage depth and the GC content of the reference were calculated using custom Python scripts. All statistics were generated after removing PCR and optical duplicates and mapped reads with Phred mapping quality $\leq 20$, meaning that any mapped reads that were not uniquely aligned were excluded from the downstream analysis. To evaluate the proportion of reads mapping to the tick vector raw sequence reads were mapped to the $I$. scapularis draft genome assembly $[16,17,37]$, using the same pipeline.

SNPs were identified using SAMtools (v. 0.1.19) [77] with the default parameters. SNP filtering was implemented in the vcftools program [78] to include only those variants meeting specific criteria: minimum SNP Phred Quality 30, minimum read depth $\geq 10$, and insertion and deletions were excluded. All filtered homozygous SNPs were annotated and the effect of SNPs on coding sequence was predicted using SnpEff v2.0b [79] using the B. burgdorferi B31 genome annotation as a reference.

\section{Statistical analysis}

We used univariate logistic regression to detect correlates of $B$. burgdorferi capture efficiency, the proportion of sequence reads derived from B. burgdorferi. We used a quasi-binomial model, allowing for flexibility in the dispersion parameter, and excluded samples identified as regression outliers using DFBETAS, DFFITS, or diagonal elements of the hat matrix. Statistical analysis was completed in R version 3.1.1 [80].

\section{Additional files}

Additional file 1: Supplementary Information for Whole genome capture of vector-borne pathogens from mixed DNA samples: a case study of Borrelia burgdorferi.

Additional file 2: Table S2AB. Mapping statistics for B. burgdorferi circular and linear plasmids.

\section{Abbreviations \\ Bp: Base pair; qPCR: Quantitative polymerase chain reaction; MLST: Multi-locus sequence typing; WGS: Whole genome sequence; SNP: Single nucleotide polymorphism; GWAS: Genome-wide association studies; VBZ: Vector-borne and zoonotic diseases.}

\section{Competing interests}

The authors declare that they have no competing interests.

\section{Authors' contributions}

GC, KW, MD, AC conceived the study; SJB, AGH, KW conducted DNA extraction and pathogen screening/typing; GC, KW performed bioinformatic analyses; GC and KW wrote the manuscript. AC, MD supervised the project and edit the manuscript. All authors read and approved the manuscript for publication.

\section{Acknowledgements}

This project was funded by the National Science Foundation/National Institutes of Health Ecology and Evolution of Infectious Diseases Program award R01 GM105246-01 (M.D.). G.C. was supported by Gaylord Donnelley Postdoctoral Environmental Fellowship (the Yale Institute for Biospheric Studies). K.S.W. was supported by the $\mathrm{NIH}$ pre-doctoral training grant 5T32Al007404-23. The authors thank Kaya Bilguvar of the Yale Center for Genomic Analysis for consultation and technical suggestions.

\section{Author details}

'Department of Epidemiology of Microbial Diseases, Yale School of Public Health, 06520 New Haven, CT, USA. ${ }^{2}$ Robinson Research Institute, University of Adelaide, 5005 Adelaide, SA, Australia. ${ }^{3}$ The Geisel School of Medicine, Dartmouth College, 03755 Hanover, NH, USA. ${ }^{4}$ Department of Ecology, Evolution and Environmental Biology, Columbia University, 10027 New York, NY, USA. ${ }^{5}$ Department of Ecology and Evolutionary Biology, Yale University, 06520 New Haven, CT, USA.

Received: 11 November 2014 Accepted: 18 May 2015

Published online: 06 June 2015

\section{References}

1. Harris SR, Feil EJ, Holden MTG, Quail MA, Nickerson EK, Chantratita N, et al. Evolution of MRSA during hospital transmission and intercontinental spread. Science. 2010:327:469-74

2. Vogler AJ, Birdsell D, Price LB, Bowers JR, Beckstrom-Sternberg SM, Auerbach RK, et al. Phylogeography of Francisella tularensis: global expansion of a highly fit clone. J Bacteriol. 2009;191:2474-84.

3. Vogler AJ, Chan F, Wagner DM, Roumagnac P, Lee J, Nera R, et al. Phylogeography and molecular epidemiology of Yersinia pestis in Madagascar. PLoS Negl Trop Dis. 2011:5:e1319.

4. Vogler AJ, Birdsell DN, Lee J, Vaissaire J, Doujet CL, Lapalus M, et al. Phylogeography of Francisella tularensis ssp. holarctica in France. Lett Appl Microbiol. 2011:52:177-80.

5. Jones KE, Patel NG, Levy MA, Storeygard A, Balk D, Gittleman JL, et al. Global trends in emerging infectious diseases. Nature. 2008:451:990-3.

6. Sistrom M, Evans B, Bjornson R, Gibson W, Balmer O, Mäser P, et al. Comparative genomics reveals multiple genetic backgrounds of human pathogenicity in the Trypanosoma brucei complex. Genome Biol Evol. 2014;6:2811-9.

7. Mu J, Myers RA, Jiang H, Liu S, Ricklefs S, Waisberg M, et al. Plasmodium falciparum genome-wide scans for positive selection, recombination hot spots and resistance to antimalarial drugs. Nat Genet. 2010;42:268-71.

8. Volkman SK, Sabeti PC, DeCaprio D, Neafsey DE, Schaffner SF, Milner DA, et al. A genome-wide map of diversity in Plasmodium falciparum. Nat Genet. 2007;39:113-9

9. Ariey F, Witkowski B, Amaratunga C, Beghain J, Langlois A-C, Khim N, et al, A molecular marker of artemisinin-resistant Plasmodium falciparum malaria. Nature. 2014:505:50-5.

10. Echodu R, Sistrom M, Bateta R, Murilla G, Okedi L, Aksoy S, et al. Genetic diversity and population structure of Trypanosoma brucei in Uganda: implications for the epidemiology of sleeping sickness and nagana. PLoS Negl Trop Dis. 2015;87:248.

11. Carpi G, Cagnacci F, Wittekindt NE, Zhao F, Qi J, Tomsho LP, et al. Metagenomic profile of the bacterial communities associated with /xodes ricinus ticks. PLoS One. 2011:6:e25604. doi:10.1371/journal.pone.0025604.

12. Oyola SO, Gu Y, Manske M, Otto TD, O'Brien J, Alcock D, et al. Efficient depletion of host DNA contamination in malaria clinical sequencing. J Clin Microbiol. 2013;51:745-51.

13. Luikart G, England PR, Tallmon D, Jordan S, Taberlet P. The power and promise of population genomics: from genotyping to genome typing. Nat Rev Genet. 2003:4:981-94.

14. Schutzer SE, Fraser-Liggett CM, Casjens SR, Qiu W-G, Dunn JJ, Mongodin EF, et al. Whole-genome sequences of thirteen isolates of Borrelia burgdorferi. J Bacteriol. 2011:193:1018-20.

15. Fraser CM, Casjens S, Huang WM, Sutton GG, Clayton R, Lathigra R, et al. Genomic sequence of a Lyme disease spirochaete, Borrelia burgdorferi. Nature. 1997;390:580-6.

16. Lawson D, Arensburger P, Atkinson P, Besansky NJ, Bruggner RV, Butler R et al. VectorBase: a data resource for invertebrate vector genomics. Nucleic Acids Res. 2009:37:D583-7.

17. Megy K, Emrich SJ, Lawson D, Campbell D, Dialynas E, Hughes DST, et al. VectorBase: improvements to a bioinformatics resource for invertebrate vector genomics. Nucleic Acids Res. 2012;40:D729-34 
18. Xu G, Wesker J, White C, Campbell J, Reich NG, Rich SM. Detection of heterogeneity of Borrelia burgdorferi in Ixodes ticks by culture-dependent and culture-independent methods. J Clin Microbiol. 2013;51:615-7.

19. Leichty A, Brisson D. Selective whole genome amplification for resequencing target microbial species from complex natural samples. Genetics. 2014;198(2):473-81.

20. Lasken RS, Stockwell TB. Mechanism of chimera formation during the multiple displacement amplification reaction. BMC Biotechnol. 2007;7:19.

21. Maricic T, Whitten M, Pääbo S. Multiplexed DNA sequence capture of mitochondrial genomes using PCR products. PLoS One. 2010;5:e14004. doi:10.1371/journal.pone.0014004

22. Meyerhans A, Vartanian J-P, Wain-Hobson S. DNA recombination during PCR. Nucleic Acids Res. 1990;18:1687-91.

23. Estrada-Peña A, Ayllón N, de la Fuente J. Impact of climate trends on tickborne pathogen transmission. Front Physiol. 2012;3:64.

24. Margos G, Vollmer SA, Ogden NH, Fish D. Population genetics, taxonomy, phylogeny and evolution of Borrelia burgdorferi sensu lato. Infect Genet Evol. 2011;11:1545-63.

25. Hoen AGA, Margos G, Bent SJ, Diuk-Wasser MA, Barbour A, Kurtenbach K et al. Phylogeography of Borrelia burgdorferi in the eastern United States reflects multiple independent Lyme disease emergence events. Proc Nat Acad Sci U S A. 2009;106:15013-8.

26. Margos G, Gatewood AG, Aanensen DM, Hanincová K, Vollmer SA, Cornet $M$, et al. MLST of housekeeping genes captures geographic population structure and suggests a European origin of Borrelia burgdorferi. Proc Natl Acad Sci U S A. 2008;105:8730-5.

27. Margos G, Tsao Jl, Castillo-Ramírez S, Girard YA, Hamer SA, Hoen AG, et al. Two boundaries separate Borrelia burgdorferi populations in North America. Appl Environ Microbiol. 2012;78:6059-67.

28. Hodges E, Xuan Z, Balija V, Kramer M, Molla MN, Smith SW, et al. Genomewide in situ exon capture for selective resequencing. Nat Genet. 2007;39:1522-7.

29. Ng SB, Turner EH, Robertson PD, Flygare SD, Abigail W, Lee C, et al. Targeted capture and massively parallel sequencing of twelve human exomes. Nature. 2010;461:272-6.

30. Hosono S, Faruqi A, Dean F, Du Y. Unbiased whole-genome amplification directly from clinical samples. Genome Res. 2003;13(5):954-64.

31. Kent BN, Salichos L, Gibbons JG, Rokas A, Newton ILG, Clark ME, et al. Complete bacteriophage transfer in a bacterial endosymbiont (Wolbachia) determined by targeted genome capture. Genome Biol Evol. 2011;3:209-18.

32. Melnikov A, Galinsky K, Rogov P, Fennell T, Van Tyne D, Russ C, et al. Hybrid selection for sequencing pathogen genomes from clinical samples. Genome Biol. 2011;12:R73. doi:10.1186/gb-2011-12-8-r73.

33. Teer JK, Bonnycastle LL, Chines PS, Hansen NF, Aoyama N, Swift AJ, et al. Systematic comparison of three genomic enrichment methods for massively parallel DNA sequencing. Genome Res. 2010;20:1420-31.

34. Tallon L, Silva J, Myers G, Sengamalay N, Ott S, Sadzewicz L, et al. Wholegenome capture and sequencing of obligate intracellular parasites. Abstract conference. The 14th annual Advances in Genome Biology and Technology (AGBT) meeting, Marco Island, Florida. 2013;127.

35. Krause J, Briggs AW, Kircher M, Maricic T, Zwyns N, Derevianko A, et al. A complete mtDNA genome of an early modern human from Kostenki, Russia. Curr Biol. 2010;20:231-6.

36. Wang G, Liveris D, Brei B, Falco RC, Fish D, Schwartz I, et al. Real-time PCR for simultaneous detection and quantification of Borrelia burgdorferi in fieldcollected Ixodes scapularis ticks from the Northeastern United States. Appl Environ Microbiol. 2003:69:4561-5.

37. Hill CA, Wikel SK. The Ixodes scapularis genome project: an opportunity for advancing tick research. Trends Parasitol. 2005;21:151-3.

38. Pagel Van Zee J, Geraci NS, Guerrero FD, Wikel SK, Stuart JJ, Nene VM, et al. Tick genomics: the Ixodes genome project and beyond. Int J Parasitol. 2007:37:1297-305.

39. Hill CA, Nene VM, Wikel SK. Proposal for sequencing the genome of the tick, ixodes scapularis, https://www.vectorbase.org/projects/proposalsequencing-genome-tick-ixodes-scapularis. Accessed 2 March 2015.

40. Schwartz JJ, Gazumyan A, Schwartz I. rRNA gene organization in the Lyme disease spirochete, Borrelia burgdorferi. J Bacteriol. 1992;174:3757-65.

41. Kozarewa I, Ning Z, Quail MA, Sanders MJ, Berriman M, Turner DJ. Amplification-free Illumina sequencing-library preparation facilitates improved mapping and assembly of ( $\mathrm{G}+\mathrm{C}$ )-biased genomes. Nat Methods. 2009;6:291-5
42. Kurtenbach K, Gatewood Hoen A, Bent S, Vollmer S, Ogden N, Margos G. Population biology of Lyme Borreliosis Spirochetes. In: Robinson DA, Feil E, Falush D, editors. Bacterial population genetics in infectious disease. New Jersey: Wiley; 2010. p. 217-39.

43. Brisson D, Drecktrah D, Eggers CH, Samuels DS: Genetics of Borrelia burgdorferi. Annu Rev Genet. 2012:46:515-36.

44. Balmer O, Tanner M. Prevalence and implications of multiple-strain infections. Lancet Infect Dis. 2011:11:868-78.

45. Scoles G, Papero M. A relapsing fever group spirochete transmitted by Ixodes scapularis ticks. Vector Borne Zoonotic Dis. 2001;1:21-34.

46. Barbour AAG, Bunikis J, Travinsky B, Hoen AG, Diuk-Wasser MA, Fish D, et al. Niche partitioning of Borrelia burgdorferi and Borrelia miyamotoi in the same tick vector and mammalian reservoir species. Am J Trop Med Hyg. 2009:81:1120-31.

47. Spielman A, Wilson M. Ecology of Ixodes dammini-borne human babesiosis and Lyme disease. Annu Rev Entomol. 1985;30:439-60.

48. Telford SR, Dawson JE, Katavolos P, Warner CK, Kolbert CP, Persing DH. Perpetuation of the agent of human granulocytic ehrlichiosis in a deer tickrodent cycle. Proc Natl Acad Sci U S A. 1996;93(June):6209-14.

49. Dupuis AP, Peters RJ, Prusinski MA, Falco RC, Ostfeld RS, Kramer LD. Isolation of deer tick virus (Powassan virus, lineage II) from Ixodes scapularis and detection of antibody in vertebrate hosts sampled in the Hudson Valley, New York State. Parasit Vectors. 2013;6:185.

50. Andersson M, Scherman K, Råberg L. Multiple-strain infections of Borrelia afzelii: a role for within-host interactions in the maintenance of antigenic diversity? Am Nat. 2013;181:545-54.

51. Cohen T, van Helden PD, Wilson D, Colijn C, McLaughlin MM, Abubakar I, et al. Mixed-strain Mycobacterium tuberculosis infections and the implications for tuberculosis treatment and control. Clin Microbiol Rev. 2012;25:708-19.

52. Alam U, Hyseni C, Symula RE, Brelsfoard C, Wu Y, Kruglov O, et al. Implications of microfauna-host interactions for trypanosome transmission dynamics in Glossina fuscipes fuscipes in Uganda. Appl Environ Microbiol. 2012;78:4627-37

53. Aksoy S, Rio RVM. Interactions among multiple genomes: tsetse, its symbionts and trypanosomes. Insect Biochem Mol Biol. 2005;35:691-8.

54. Aksoy S, Caccone A, Galvani AP, Okedi LM. Glossina fuscipes populations provide insights for human African trypanosomiasis transmission in Uganda. Trends Parasitol. 2013;29:394-406.

55. Garcia E, Azambuja P. Development and interactions of Trypanosoma cruzi within the insect vector. Parasitol Today. 1991;7:240-4.

56. Calvignac-Spencer S, Leendertz SAJ, Gillespie TR, Leendertz FH. Wild great apes as sentinels and sources of infectious disease. Clin Microbiol Infect. 2012:18:521-7.

57. Liu W, Li Y, Shaw KS, Learn GH, Plenderleith LJ, Malenke JA, et al. African origin of the malaria parasite Plasmodium vivax. Nat Commun. 2014:5:3346.

58. Reed PE, Mulangu S, Cameron KN, Ondzie AU, Joly D, Bermejo M, et al. A new approach for monitoring ebolavirus in wild great apes. PLoS Negl Trop Dis. 2014;8:e3143. doi:10.1371/journal.pntd.0003143.

59. Taberlet $\mathrm{P}$, Coissac E, Hajibabaei M, Rieseberg LH. Environmental DNA. Mo Ecol. 2012;21:1789-93.

60. Alex Buerkle C, Gompert Z, Buerkle AC, Gompert Z. Population genomics based on low coverage sequencing: how low should we go? Mol Ecol. 2013;22(11):3028-35.

61. Mason KL, Stepien TA, Blum JE, Holt JF, Labbe NH, Rush JS, et al. From commensal to pathogen: translocation of Enterococcus faecalis from the midgut to the hemocoel of Manduca sexta. MBio. 2011; 17;2(3):e00065-11. doi: 10.1128/mBio.00065-11.

62. Mongodin EF, Casjens SR, Bruno JF, Xu Y, Drabek EF, Riley DR, et al. Interand intra-specific pan-genomes of Borrelia burgdorferi sensu lato: genome stability and adaptive radiation. BMC Genomics. 2013;14:693.

63. Falco RC, Fish D. A comparison of methods for sampling the deer tick, Ixodes dammini, in a Lyme disease endemic area. Exp Appl Acarol. 1992;14:165-73.

64. Keirans JE, Hutcheson HJ, Durden LA, Klompen JS. Ixodes (Ixodes) scapularis (Acari:Ixodidae): redescription of all active stages, distribution, hosts, geographical variation, and medical and veterinary importance. J Med Entomol. 1996:33:297-318.

65. Norris DE, Klompen JS, Keirans JE, Black WC. Population genetics of Ixodes scapularis (Acari: Ixodidae) based on mitochondrial 165 and 125 genes. J Med Entomol. 1996;33:78-89.

66. Beati L, Keirans JE. Analysis of the systematic relationships among ticks of the genera Rhipicephalus and Boophilus (Acari: Ixodidae) based on 
mitochondrial 125 ribosomal DNA gene sequences and morphological characters. J Parasitol. 2001;87:32-48.

67. Tsao J, Wootton J, Bunikis J, Luna M, Fish D, Barbour A. An ecological approach to preventing human infection: vaccinating wild mouse reservoirs intervenes in the Lyme disease cycle. Proc Natl Acad Sci U S A. 2004;101:18159-64

68. Roche NimbleGen. SeqCap EZ library SR user's guide version 4.2. Roche NimbleGen, Inc. Madison, WI. 2013.

69. Baillie JK, Barnett MW, Upton KR, Gerhardt DJ, Richmond TA, De Sapio F, et al. Somatic retrotransposition alters the genetic landscape of the human brain. Nature. 2011:479:534-7.

70. Barbour A, Travinsky B. Evolution and distribution of the ospC gene, a transferable serotype determinant of Borrelia burgdorferi. MBio. 2010;1:e00153-10

71. Pal U, Yang X, Chen M, Bockenstedt LK, Anderson JF, Flavell RA, et al. OspC facilitates Borrelia burgdorferi invasion of Ixodes scapularis salivary glands. J Clin Invest. 2004:113:220-30.

72. Gilmore RD, Piesman J. Inhibition of Borrelia burgdorferi migration from the midgut to the salivary glands following feeding by ticks on OspCimmunized mice. Infect Immun. 2000;68:411-4.

73. Marconi RT, Samuels DS, Garon CF. Transcriptional analyses and mapping of the ospC gene in Lyme disease spirochetes. J Bacteriol. 1993;175:926-32.

74. Qiu W-G, Dykhuizen DE, Acosta MS, Luft BJ. Geographic uniformity of the Lyme disease spirochete (Borrelia burgdorferi) and its shared history with tick vector (Ixodes scapularis) in the Northeastern United States. Genetics. 2002;160:833-49.

75. Wang IN, Dykhuizen DE, Qiu W, Dunn JJ, Bosler EM, Luft BJ. Genetic diversity of ospC in a local population of Borrelia burgdorferi sensu stricto. Genetics. 1999;151:15-30.

76. Li H, Durbin R. Fast and accurate short read alignment with BurrowsWheeler transform. Bioinformatics. 2009;25:1754-60.

77. Li H, Handsaker B, Wysoker A, Fennell T, Ruan J, Homer N, et al. The Sequence Alignment/Map format and SAMtools. Bioinformatics. 2009;25:2078-9.

78. Danecek P, Auton A, Abecasis G, Albers CA, Banks E, DePristo MA, et al. The variant call format and VCFtools. Bioinformatics. 2011;27:2156-8.

79. Cingolani $P$, Platts $A$, Wang $L L$, Coon M, Nguyen T, Wang L, et al. SNPs in the genome of Drosophila melanogaster strain w1118; iso-2; iso-3. Fly (Austin). 2012;6:80-92.

80. R Core. R: a language and environment for statistical computing. R Foundation for Statistical Computing, Vienna, Austria. 2014.

\section{Submit your next manuscript to BioMed Central and take full advantage of:}

- Convenient online submission

- Thorough peer review

- No space constraints or color figure charges

- Immediate publication on acceptance

- Inclusion in PubMed, CAS, Scopus and Google Scholar

- Research which is freely available for redistribution 